\title{
MODIFIED WIENER EQUATIONS
}

\author{
WILL WATKINS
}

(Received 16 January 2001)

To the memory of the late Prof Joseph Wiener

\begin{abstract}
This paper is concerned with a class of functional differential equations whose argument transforms are involutions. In contrast to the earlier works in this area, which have used only involutions with a fixed point, we also admit involutions without a fixed point. In the first case, an initial value problem for a differential equation with involution is reduced to an initial value problem for a higher order ordinary differential equation. In our case, either two initial conditions or two boundary conditions are necessary for a solution; the equation is then reduced to a boundary value problem for a higher order ODE.
\end{abstract}

2000 Mathematics Subject Classification. 34K05, 34K10.

1. Introduction. When studying the general properties of functional differential equations, it is always important to find and solve selected classes of equations as explicitly as possible, using methods that are capable of generalization. Differential equations with involutions is one of those classes.

A function $f(x) \not \equiv x$, that maps a set, $G$, of real numbers onto itself and satisfies on $G$ the condition

$$
f(f(x))=x \quad \text { or } \quad f^{-1}(x)=f(x),
$$

is called an involution on $G$. The simplest examples of involutions are

$$
\begin{array}{ll}
f(x)=-x & \text { or } \quad f(x)=c-x, \quad x \in(-\infty, \infty), c \in \mathbb{R}, \\
f(x)=\frac{1}{x} \quad \text { or } & f(x)=-\frac{1}{x}, \quad x \in(-\infty, 0) \cup(0, \infty) .
\end{array}
$$

DEFINITION 1.1. A relation of the form

$$
F\left(x, y\left(f_{1}(x)\right), \ldots, y\left(f_{m}(x)\right), \ldots, y^{(n)}\left(f_{1}(x)\right), \ldots, y^{(n)}\left(f_{m}(x)\right)\right)=0,
$$

where $f_{i}\left(f_{i}(x)\right)=x$ for every $i$, and $f_{i}(x) \not \equiv x$ for some $i$, is called a differential equation with involutions.

We investigate differential equations of the form

$$
y^{\prime}(x)=F(x, y(x), y(f(x)))
$$

where $f(x)$ is an involution. The properties of involutions that are key to our discussion are the following. 
ProperTy 1.2. Suppose that $f(x)$ is a continuous involution on an open connected set, $G$. Then $f$ has a unique fixed point on $G$, and $f$ is decreasing on $G$.

Proof. The function $f$ is monotone, one-to-one, and continuous on $G$, consequently $\Phi(x)=f(x)-x$ is monotone and continuous. Since $f(x) \neq \equiv$, there exists $x_{0}$ such that $f\left(x_{0}\right) \neq x_{0}$. If $x_{0}<f\left(x_{0}\right)$, then $\Phi\left(x_{0}\right)>0$ and $\Phi\left(f\left(x_{0}\right)\right)<0$, hence there is an $x_{1} \in\left(x_{0}, f\left(x_{0}\right)\right)$ such that $\Phi\left(x_{1}\right)=0$. A similar argument holds when $f\left(x_{0}\right)<x_{0}$. Therefore, there is a unique fixed point. Suppose $f$ is increasing on $G$ and $f\left(x_{0}\right)<x_{0}$, then $f\left(f\left(x_{0}\right)\right)<f\left(x_{0}\right)$ or $x_{0}<f\left(x_{0}\right)$, a contradiction. Similarly if $f\left(x_{0}\right)>x_{0}$, then $f\left(f\left(x_{0}\right)\right)>f\left(x_{0}\right)$ or $x_{0}>f\left(x_{0}\right)$, again a contradiction.

DEFINITION 1.3. If $x \in \mathscr{D}$, where $\mathscr{D}$ is the domain of an involution $f$, then the component $\mathscr{K}(x)$ of $\mathscr{D}$ is the maximally connected subset of $\mathscr{D}$ that contains $x$. Two components, $\mathscr{K}\left(x_{1}\right)$ and $\mathscr{K}\left(x_{2}\right)$, are said to be $f$-complements if $f\left(\mathscr{K}\left(x_{1}\right)\right)=\mathscr{K}\left(x_{2}\right)$.

It is observed that $f(\mathscr{K}(x))=\mathscr{K}(f(x))$. Furthermore, whenever $f\left(x_{1}\right)=x_{2}$, then $\mathscr{K}\left(x_{1}\right)$ and $\mathscr{K}\left(x_{2}\right)$ are $f$-complements. The following statement is a corollary of Property 1.2. It highlights the fact that when $f$ has no fixed point, the $f$-complements are disjoint. This is a key idea in understanding the theorems that follow.

ProperTy 1.4. Suppose that $f(x)$ is a continuous involution on $\mathscr{D} \subseteq \mathbb{R}$, and $f$ has no fixed point. Then, for $x_{0} \in \mathscr{D}, f\left(\mathscr{K}\left(x_{0}\right)\right) \cap \mathscr{K}\left(x_{0}\right)$ is empty.

A consequence of Properties 1.2 and 1.4 is that the domain of an increasing involution is not connected.

The study of differential equations with involution was initiated by Wiener in $[12,13]$ and continued by many authors. In [8], Przeworska-Rolewicz developed an algebraic approach to equations with transformed arguments, including involutions. Castelan and Infante [3] used the solutions of differential equations with involutions in the construction of Liapunov functionals to explore the stability of retarded differential equations. Šharkovskiǔ [9] investigated functional differential equations with a finite group of argument transformations and carried out, in particular, a geometric analysis of the equation with reflection of the argument,

$$
y^{\prime}(x)=F(y(x), y(-x)) .
$$

Letting $y(-x)=z(x)$ he obtained

$$
\frac{d z}{d x}=-y^{\prime}(-x)=-F(y(-x), y(x))=-F(z(x), y(x)) .
$$

Hence, the solutions of (1.5) correspond to the solutions of the system of ordinary differential equations

$$
\frac{d y}{d x}=F(y, z), \quad \frac{d z}{d x}=-F(z, y)
$$

with the condition $y(0)=z(0)$. From the qualitative analysis of the solutions of the system he derived qualitative information about the solutions of the equation with reflection. Shevelo and Gritsai [10] considered a class of differential equations with involutions and studied some of their properties, especially the boundedness of solutions on $[0, \infty)$. The existence of a unique bounded solution of the equation with 
reflection of the argument,

$$
y^{\prime}(x)=F(x, y(x), y(-x)), \quad x \in \mathbb{R}
$$

has been studied by Aftabizadeh et al. [1]. Boundary value problems for differential equations with reflection have been explored by Wiener and Aftabizadeh [15] and their results have been generalized by Gupta [5, 6, 7]. Differential equations with involutions can be transformed by differentiation to higher order ordinary differential equations. Therefore they belong to the class of reducible functional differential equations. Busenberg and Travis [2] used reducible functional differential equations in the study of certain biological models. For further references see Wiener [14]. Recently, pantograph equations with involution have been introduced by Derfel and Iserles [4].

2. Main results and examples. The following theorems generalize the corresponding results of $[12,13]$ (see also [14]).

THEOREM 2.1. Let the initial value problem

$$
y^{\prime}(x)=F(x, y(x), y(f(x))), \quad y\left(x_{0}\right)=y_{0},
$$

satisfy the following hypotheses:

(1) The function $f(x)$ is a continuously differentiable involution on its domain $\mathscr{D}$. Furthermore, $f\left(\mathscr{K}\left(x_{0}\right)\right)=\mathscr{K}\left(x_{0}\right)=\mathscr{D}$, where $\mathscr{K}\left(x_{0}\right)$ is the $f$-complement of $x_{0}$.

(2) The function $F$ is defined and is continuously differentiable in the whole space of its arguments, that is, on $\mathscr{D} \times y(\mathscr{D}) \times y(f(\mathscr{D}))$.

(3) Equation (2.1) is uniquely solvable with respect to $y(f(x))$ :

$$
y(f(x))=G\left(x, y(x), y^{\prime}(x)\right) .
$$

Then the solution of the ordinary differential equation

$$
y^{\prime \prime}(x)=\frac{\partial F}{\partial x}+\frac{\partial F}{\partial y(x)} y^{\prime}(x)+\frac{\partial F}{\partial y(f(x))} f^{\prime}(x) F(f(x), y(f(x)), y(x)),
$$

where $y(f(x))$ is given by the expression (2.2), with the boundary conditions,

$$
y\left(x_{0}\right)=y_{0}, \quad y^{\prime}\left(f\left(x_{0}\right)\right)=F\left(f\left(x_{0}\right), y\left(f\left(x_{0}\right)\right), y_{0}\right), \quad x_{0} \in \mathscr{D},
$$

is a solution of (2.1) on $\mathscr{D}$.

Proof. Differentiating (2.1) gives

$$
y^{\prime \prime}(x)=\frac{\partial F}{\partial x}+\frac{\partial F}{\partial y(x)} y^{\prime}(x)+\frac{\partial F}{\partial y(f(x))} f^{\prime}(x) y^{\prime}(f(x)) .
$$

But from (2.1) and the relation $f(f(x))=x$, it follows that

$$
y^{\prime}(f(x))=F(f(x), y(f(x)), y(x)) .
$$

The second boundary condition (2.4) comes from setting $x=f\left(x_{0}\right)$ in (2.1); this is an implicit compatible condition. 
In Theorem 2.1, $f$ must have a fixed point by Property 1.2. If $x_{0}$ is the fixed point of $f$, then the boundary conditions (2.4) become initial conditions,

$$
y\left(x_{0}\right)=y_{0}, \quad y^{\prime}\left(x_{0}\right)=F\left(x_{0}, y_{0}, y_{0}\right), \quad x_{0} \in \mathscr{D} .
$$

This is Wiener's theorem [12].

Earlier works in this area study cases where the involution has a fixed point, identify initial conditions at that fixed point and find solutions on the connected domain. We generalize in two ways, first by considering initial conditions at points different than the fixed point, and second by considering disjoint domains. The next theorem is a special case of Theorem 2.1.

THEOREM 2.2. Assume that in the problem

$$
y^{\prime}(x)=F(y(f(x))), \quad y\left(x_{0}\right)=y_{0},
$$

the function $f(x)$ is a continuously differentiable involution on its connected domain, D; and the function $F$ is defined, continuously differentiable, and strictly monotonic on $y(\mathscr{D})$. Then the solution of the ordinary differential equation

$$
y^{\prime \prime}(x)=F^{\prime}(y(f(x))) F(y(x)) f^{\prime}(x), \quad y(f(x))=F^{-1}\left(y^{\prime}(x)\right)
$$

with the boundary conditions

$$
y\left(x_{0}\right)=y_{0}, \quad y^{\prime}\left(f\left(x_{0}\right)\right)=F\left(y_{0}\right),
$$

is a solution of (2.8).

EXAMPLE 2.3. Silberstein [11] solved the equation

$$
y^{\prime}(x)=y\left(\frac{1}{x}\right), \quad 0<x<\infty,
$$

by assuming a solution of the form

$$
y(x)=x^{k}+\lambda x^{m}
$$

where $k, m$, and $\lambda$ are constants. Wiener [12] obtained a solution by differentiating (2.11),

$$
y^{\prime \prime}(x)=-\frac{1}{x^{2}} y^{\prime}\left(\frac{1}{x}\right)=-\frac{1}{x^{2}} y(x),
$$

whence,

$$
x^{2} y^{\prime \prime}(x)+y(x)=0 .
$$

This is a Cauchy-Euler equation, with the general solution of the form

$$
y(x)=A_{1} x^{r_{1}}+A_{2} x^{r_{2}} .
$$

Hence $r_{1}$ and $r_{2}$ are the roots of

$$
r^{2}-r+1=0
$$


Suppose we seek a solution of Silbertein's equation (2.11) satisfying the initial condition

$$
y\left(x_{0}\right)=y_{0}
$$

Since (2.11) implies that $y^{\prime}(1 / x)=y(x)$, we have the additional initial condition

$$
y^{\prime}\left(\frac{1}{x_{0}}\right)=y_{0}
$$

Therefore $A_{1}$ and $A_{2}$ are uniquely determined by the system of equations,

$$
x_{0}^{r_{1}} A_{1}+x_{0}^{r_{2}} A_{2}=y_{0}, \quad r_{1} x_{0}^{r_{2}} A_{1}+r_{2} x_{0}^{r_{1}} A_{2}=y_{0} .
$$

In contrast to the previous example where $f$ has a connected domain, the next example considers a disconnected domain. By modifying the domain in Silberstein's problem we begin to see the significance of a disconnected domain.

EXAMPLE 2.4. Consider the differential equation

$$
y^{\prime}(x)=y(f(x)), \quad f(x)=\frac{1}{x}, x \in(0,1) \cup(1, \infty),
$$

with the initial conditions

$$
y(2)=y_{2}, \quad y^{\prime}(2)=\tilde{y}_{2} .
$$

It is important that the two components of the domain are $f$-complements. That means that compatible conditions generated from the initial conditions are in the other component and hence do not affect the continuity of the solution in the original component. Therefore, we seek a solution of the form

$$
y(x)= \begin{cases}C_{1} x^{r_{1}}+C_{2} x^{r_{2}}, & x \in(0,1), \\ D_{1} x^{r_{1}}+D_{2} x^{r_{2}}, & x \in(1, \infty) .\end{cases}
$$

The initial conditions uniquely determine $D_{1}$ and $D_{2}$ by the system of equations

$$
y(2)=y_{2}=2^{r_{1}} D_{1}+2^{r_{2}} D_{2}, \quad y^{\prime}(2)=\tilde{y}_{2}=r_{1} 2^{-r_{2}} D_{1}+r_{2} 2^{-r_{1}} D_{2} .
$$

The determinant of this system is $r_{2}-r_{1}$ hence there is a unique solution for $D_{1}$ and $D_{2}$. The differential equation implies that $y^{\prime}(1 / x)=y(x)$, therefore the initial conditions in (2.21) generate compatible conditions

$$
y\left(\frac{1}{2}\right)=\tilde{y}_{2}, \quad y^{\prime}\left(\frac{1}{2}\right)=y_{2},
$$

which produce the system of equations

$$
y\left(\frac{1}{2}\right)=\tilde{y}_{2}=2^{-r_{1}} C_{1}+2^{-r_{2}} C_{2}, \quad y^{\prime}\left(\frac{1}{2}\right)=y_{2}=r_{1} 2^{r_{2}} C_{1}+r_{2} 2^{r_{1}} C_{2},
$$

which also has a unique solution.

This last example is rather contrived because the involution could be extended to a continuous involution on a connected domain. It does, however, emphasize the 
significance of the connectedness of the domain $f$, showing that the disconnected domain admits solutions that the connected domain does not. In the case that $\mathscr{K}\left(x_{0}\right)$ is its own $f$-complement, the solution of the differential equation is a continuous function on a connected set. One initial condition generates a second compatible boundary condition (or a second initial condition in the case that $x_{0}$ is a fixed point). The original condition and the compatible condition then determine the coefficients in the solution of the second order ODE. In contrast, when the domain of $f$ is the union of two disjoint compatible components, two initial conditions are necessary, because the generated compatible conditions are in the other component and we need two conditions in each component to determine a solution. The following theorems clarify the idea.

THEOREM 2.5. Let the initial value problem

$$
y^{\prime}(x)=F(x, y(x), y(f(x))), \quad y\left(x_{0}\right)=y_{0}, \quad y^{\prime}\left(x_{0}\right)=\tilde{y}_{0},
$$

satisfy the following hypotheses:

(1) The function $f(x)$ is a continuously differentiable involution on its domain, $\mathscr{D}$. Furthermore, $f\left(\mathscr{K}\left(x_{0}\right)\right) \cup \mathscr{K}\left(x_{0}\right)=\mathscr{D}$ and $f\left(\mathscr{K}\left(x_{0}\right)\right) \cap \mathscr{K}\left(x_{0}\right)$ is empty.

(2) The function $F$ is defined and is continuously differentiable in the whole space of its arguments, that is, on $\mathscr{D} \times y(\mathscr{D}) \times y(f(\mathscr{D}))$.

(3) Equation (2.26) is uniquely solvable with respect to $y(f(x))$ :

$$
y(f(x))=G\left(x, y(x), y^{\prime}(x)\right) .
$$

Then the solution of the ordinary differential equation

$$
y^{\prime \prime}(x)=\frac{\partial F}{\partial x}+\frac{\partial F}{\partial y(x)} y^{\prime}(x)+\frac{\partial F}{\partial y(f(x))} f^{\prime}(x) F(f(x), y(f(x)), y(x)),
$$

where $y(f(x))$ is given by the expression (2.27), with the boundary conditions

$$
\begin{gathered}
y\left(x_{0}\right)=y_{0}, \quad y^{\prime}\left(x_{0}\right)=\tilde{y}_{0}, \\
y^{\prime}\left(f\left(x_{0}\right)\right)=F\left(f\left(x_{0}\right), y\left(f\left(x_{0}\right)\right), y_{0}\right), \quad y\left(f\left(x_{0}\right)\right)=G\left(x_{0}, y_{0}, \tilde{y}_{0}\right),
\end{gathered}
$$

is a solution of problem (2.26) on $\mathscr{D}$.

PROoF. The assertion follows from the arguments of Theorem 2.1. Setting $x=$ $f\left(x_{0}\right)$ in (2.6) we obtain

$$
y^{\prime}\left(f\left(x_{0}\right)\right)=F\left(f\left(x_{0}\right), y\left(f\left(x_{0}\right)\right), y_{0}\right) .
$$

The other compatibility condition in (2.29) is obtained by setting $x=x_{0}$ in (2.27).

As a special case consider the following theorem.

THEOREM 2.6. Assume that in the initial value problem

$$
y^{\prime}(x)=F(y(f(x))), \quad y\left(x_{0}\right)=y_{0}, \quad y^{\prime}\left(x_{0}\right)=\tilde{y}_{0},
$$

the function $f(x)$ is a continuously differentiable involution on its domain, $\mathscr{D} ; f(\mathscr{K}(x)) \cup$ $\mathscr{K}(x)=\mathscr{D} ; f(\mathscr{K}(x)) \cap \mathscr{K}(x)$ is empty; and the function $F$ is defined, continuously differentiable, and strictly monotonic on $y(\mathscr{D})$. Then the solution of the ordinary differential 
equation

$$
y^{\prime \prime}(x)=F^{\prime}(y(f(x))) F(y(x)) f^{\prime}(x), \quad y(f(x))=F^{-1}\left(y^{\prime}(x)\right)
$$

with the initial conditions

$$
\begin{array}{cl}
y\left(x_{0}\right)=y_{0}, & y^{\prime}\left(x_{0}\right)=\tilde{y}_{0}, \\
y^{\prime}\left(f\left(x_{0}\right)\right)=F\left(y_{0}\right), & y\left(f\left(x_{0}\right)\right)=F^{-1}\left(\tilde{y}_{0}\right),
\end{array}
$$

is a solution of problem (2.31).

EXAMPLE 2.7. The involution in this example is strictly increasing and has a domain which is the union of two complementary components,

$$
\mathscr{D}=(-\infty, 0) \cup(0, \infty) .
$$

Furthermore, there is no way to extend the involution to a continuous function on a connected domain (which is always the case with an increasing involution). The equation

$$
y^{\prime}(x)=\operatorname{ay}\left(-\frac{1}{x}\right)
$$

involves an increasing involution. This transforms to

$$
y^{\prime \prime}(x)=\frac{a^{2}}{x^{2}} y(x) \quad \text { or } \quad x^{2} y^{\prime \prime}(x)-a^{2} y(x)=0 .
$$

We look for a solution of the form $y(x)=|x|^{r}$ and obtain the characteristic equation $r^{2}-r-a^{2}=0$, with the roots

$$
r_{1}=\frac{1+\sqrt{1+4 a^{2}}}{2}, \quad r_{2}=\frac{1-\sqrt{1+4 a^{2}}}{2} .
$$

Therefore, the general solution of (2.36) is

$$
y(x)= \begin{cases}C_{1}(-x)^{r_{1}}+C_{2}(-x)^{r_{2}}, & x<0, \\ D_{1} x^{r_{1}}+D_{2} x^{r_{2}}, & x>0 .\end{cases}
$$

Furthermore, because $r_{1}+r_{2}=1$,

$$
y^{\prime}(x)= \begin{cases}-r_{2} C_{2}(-x)^{-r_{1}}-r_{1} C_{1}(-x)^{-r_{2}}, & x<0, \\ r_{2} D_{2} x^{-r_{1}}+r_{1} D_{1} x^{-r_{2}}, & x>0 .\end{cases}
$$

Note,

$$
y\left(-\frac{1}{x}\right)= \begin{cases}D_{1}(-x)^{-r_{1}}+D_{2}(-x)^{-r_{2}}, & x<0, \\ C_{1} x^{-r_{1}}+C_{2} x^{-r_{2}}, & x>0 .\end{cases}
$$

Substituting $y(x)$ into (2.35), we obtain

$$
C_{1}=-\frac{a}{r_{1}} D_{2}=\frac{r_{2}}{a} D_{2}, \quad C_{2}=-\frac{a}{r_{2}} D_{1}=\frac{r_{1}}{a} D_{1},
$$


hence,

$$
y(x)= \begin{cases}r_{2} a^{-1} D_{2}(-x)^{r_{1}}+r_{1} a^{-1} D_{1}(-x)^{r_{2}}, & x<0, \\ D_{1} x^{r_{1}}+D_{2} x^{r_{2}}, & x>0 .\end{cases}
$$

Equation (2.38) has four undetermined coefficients. We see in (2.42) that there is some "coupling" of coefficients. Since there are still two undetermined coefficients, we need either two initial conditions (as stated in the theorem) or boundary conditions that are not compatibly related. That is, $y\left(x_{0}\right)=y_{0}$ and $y^{\prime}\left(x_{0}\right)=\tilde{y}_{0}$ uniquely determine $D_{1}$ and $D_{2}$, but there are other conditions that determine the coefficients as illustrated in the next examples.

EXAMPLE 2.8. Consider a particular case of (2.35) with boundary conditions

$$
y^{\prime}(x)=\frac{\sqrt{3}}{2} y\left(-\frac{1}{x}\right), \quad y(1)=1, y(4)=1 \text {. }
$$

Then $r_{1}=3 / 2$ and $r_{2}=-1 / 2$, so when $x>0$,

$$
y(x)=D_{1} x^{3 / 2}+D_{2} x^{1 / 2} .
$$

The boundary conditions imply that $D_{1}=-1 / 6$ and $D_{2}=7 / 6$. From (2.42) the solution of $(2.43)$ is

$$
y(x)= \begin{cases}-\frac{7 \sqrt{3}}{18}(-x)^{3 / 2}-\frac{\sqrt{3}}{6}(-x)^{-1 / 2}, & x<0, \\ -\frac{1}{6} x^{3 / 2}+\frac{7}{6} x^{-1 / 2}, & x>0 .\end{cases}
$$

Boundary conditions $y(A)=y_{A}$ and $y(B)=y_{B}$ yield a unique solution of (2.35). There are three cases: either $A>B>0$, or $A<B<0$, or $A<0$ and $B>0$. In the first and second cases $D_{1}$ and $D_{2}$ can be found by using only half of (2.42). As illustrated above, if $A>B>0$, then the system

$$
A^{r_{1}} D_{1}+A^{r_{2}} D_{2}=y_{A}, \quad B^{r_{1}} D_{1}+B^{r_{2}} D_{2}=y_{B},
$$

has a unique solution. Similarly, if $A<B<0$, then

$$
\begin{aligned}
& r_{2} a^{-1}(-A)^{r_{1}} D_{2}+r_{1} a^{-1}(-A)^{r_{2}} D_{1}=y_{A}, \\
& r_{2} a^{-1}(-B)^{r_{1}} D_{2}+r_{1} a^{-1}(-B)^{r_{2}} D_{1}=y_{B},
\end{aligned}
$$

has a unique solution. In the case that $A<0$ and $B>0,(2.42)$ produces the system

$$
r_{1} a^{-1}(-A)^{r_{2}} D_{1}+r_{2} a^{-1}(-A)^{r_{1}} D_{2}=y_{A}, \quad B^{r_{1}} D_{1}+B^{r_{2}} D_{2}=y_{B},
$$

which also has a unique solution.

Care must be taken in choosing boundary conditions. The next two examples show that some choices of boundary conditions allow no solution while other choices produce an infinite number of solutions. 
EXAMPLE 2.9. The boundary value problem

$$
y^{\prime}(x)=a y\left(-\frac{1}{x}\right), \quad y(1)=y_{1}, y^{\prime}(-1)=b y_{1}, a \neq b,
$$

has no solution because the differential equation implies the compatible condition $y^{\prime}(-1)=a y(1)$.

EXAMPLE 2.10. On the other hand,

$$
y^{\prime}(x)=a y\left(-\frac{1}{x}\right), \quad y(1)=y_{1}, y^{\prime}(-1)=a y_{1},
$$

has many solutions. These (compatible) boundary conditions imply one another by the differential equation and therefore do not give sufficient information to reduce the number of coefficients.

One safe choice of boundary conditions is $y\left(x_{0}\right)=y_{0}$ and $y\left(f\left(x_{0}\right)\right)=\tilde{y}_{0}$ as illustrated in the next example.

EXAMPLE 2.11. Consider

$$
y^{\prime}(x)=\frac{\sqrt{3}}{2} y\left(-\frac{1}{x}\right), \quad y(-1)=1, y(1)=1 .
$$

Then $r_{1}=3 / 2$ and $r_{2}=-1 / 2$, and the boundary conditions applied to (2.42) gives $D_{1}=(\sqrt{3}+1) / 4$ and $D_{2}=(\sqrt{3}-3) / 4$ for the solution,

$$
y(x)= \begin{cases}\frac{\sqrt{3}-1}{4}(-x)^{3 / 2}+\frac{3+\sqrt{3}}{4}(-x)^{-1 / 2}, & x<0, \\ \frac{\sqrt{3}+1}{4} x^{3 / 2}+\frac{\sqrt{3}-3}{4} x^{-1 / 2}, & x>0 .\end{cases}
$$

EXAMPLE 2.12. The final example considers the class of equations

$$
x^{m} y^{\prime}(x)=a y\left(-\frac{1}{x}\right)
$$

with the integer $m>0$. Interestingly enough, the solutions differ depending on $m$ being even or odd. Differentiating gives

$$
x^{m} y^{\prime \prime}(x)+m x^{m-1} y^{\prime}(x)=\frac{a}{x^{2}} y^{\prime}\left(-\frac{1}{x}\right) .
$$

Replacing $x$ with $-1 / x$ in (2.54) shows that $y^{\prime}(-1 / x)=(-1)^{m} a x^{m} y(x)$ converting (2.55) to

$$
x^{2} y^{\prime \prime}(x)+m x y^{\prime}(x)-(-1)^{m} a^{2} y(x)=0,
$$

which has the characteristic equation

$$
r^{2}+(m-1) r-(-1)^{m} a^{2}=0 .
$$

In the case that $m$ is even, the roots of (2.57) are real, hence all twice-differentiable solutions of (2.54) do not oscillate. On the other hand, when $m$ is odd and $(m-1) / 2<$ $|a|$ the solutions oscillate. 
ACKNOWLEDGEMENT. This paper has its seedlings in conversation with Professor Joseph Wiener. It grew and flourished through numerous afternoon discussions. The author is most grateful for the seed, for the warm environment for growth, and for the wealth of experience and depth of understanding generously given by a kind colleague.

\section{REFERENCES}

[1] A. R. Aftabizadeh, Y. K. Huang, and J. Wiener, Bounded solutions for differential equations with reflection of the argument, J. Math. Anal. Appl. 135 (1988), no. 1, 31-37. MR 89k:34119. Zbl 655.34030.

[2] S. N. Busenberg and C. C. Travis, On the use of reducible-functional-differential equations in biological models, J. Math. Anal. Appl. 89 (1982), no. 1, 46-66. MR 84f:34102. Zbl 513.34075 .

[3] W. B. Castelan and E. F. Infante, On a functional equation arising in the stability theory of difference-differential equations, Quart. Appl. Math. 35 (1977), no. 3, 311-319. MR 58\#11772. Zbl 372.34043.

[4] G. Derfel and A. Iserles, The pantograph equation in the complex plane, J. Math. Anal. Appl. 213 (1997), no. 1, 117-132. MR 98f:34094. Zbl 891.34072.

[5] C. P. Gupta, Boundary value problems for differential equations in Hilbert spaces involving reflection of the argument, J. Math. Anal. Appl. 128 (1987), no. 2, 375-388. MR 89b:34046. Zbl 658.34053.

[6]___ Existence and uniqueness theorems for boundary value problems involving reflection of the argument, Nonlinear Anal. 11 (1987), no. 9, 1075-1083. MR 88j:34143. Zbl 632.34069.

[7] _ Two-point boundary value problems involving reflection of the argument, Int. J. Math. Math. Sci. 10 (1987), no. 2, 361-371. MR 88c:34021. Zbl 622.34015.

[8] D. Przeworska-Rolewicz, Equations with Transformed Argument. An Algebraic Approach, Modern Analytic and Computational Methods in Science and Mathematics, Elsevier Scientific Publishing \& PWN-Polish Scientific Publishers, Amsterdam \& Warsaw, 1973. MR 58\#12456. Zbl 271.47008.

[9] A. N. Šarkovskiū, Functional-differential equations with a finite group of argument transformations, Asymptotic Behavior of Solutions of Functional-Differential Equations, Coll. sci. Works, Akad. Nauk Ukrain. SSR, Inst. Mat., Kiev, 1978, pp. 118-142 (Russian). MR 80m:34071. Zbl 413.34071.

[10] V. N. Shevelo and A. G. Gritsaĭ, Some approaches to the study of the properties of solutions of differential equations with involutions, Methods for Investigating Differential and Functional-Differential Equations, Akad. Nauk Ukrain. SSR, Inst. Mat., Kiev, 1990, pp. 110-117 (Russian). MR 92j:34022.

[11] L. Silberstein, Solution of the equation $f^{\prime}(x)=f(1 / x)$, Philos. Mag. (7) 30 (1940), 185186. MR 2,134c. Zbl 026.22304.

[12] J. Wiener, Differential equations with involutions, Differencial'nye Uravnenija 5 (1969), 1131-1137 (Russian). MR 40\#1681.

[13] _ Partial differential equations with involutions, Differencial'nye Uravnenija 6 (1970), 1320-1322 (Russian). MR 42\#4904.

[14] _ Generalized Solutions of Functional-Differential Equations, World Scientific Publishing, New Jersey, 1993. MR 94m:34174. Zbl 874.34054.

[15] J. Wiener and A. R. Aftabizadeh, Boundary value problems for differential equations with reflection of the argument, Int. J. Math. Math. Sci. 8 (1985), no. 1, 151-163. MR 86i:34086. Zbl 583.34055.

Will Watkins: Department of Mathematics, University of TeXas, Pan American, EDINBURG, TX 78539, USA

E-mail address: ww7880@panam.edu 


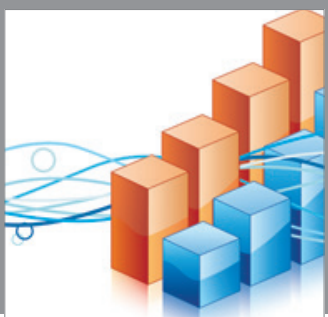

Advances in

Operations Research

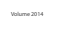

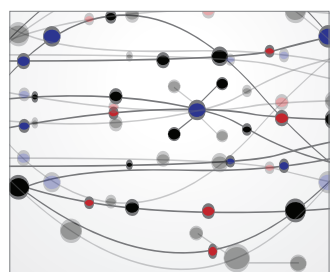

\section{The Scientific} World Journal
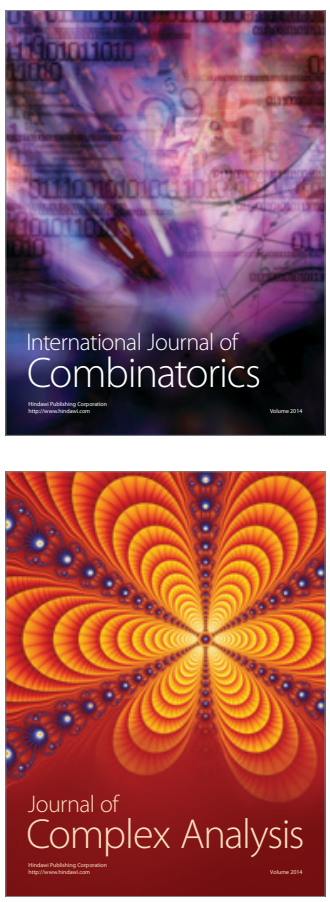

International Journal of

Mathematics and

Mathematical

Sciences
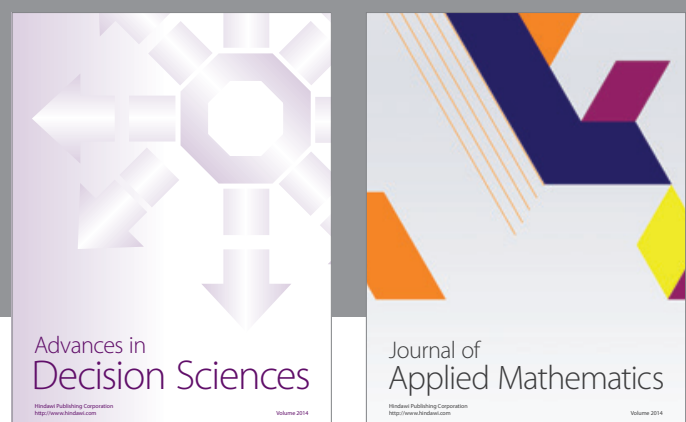

Journal of

Applied Mathematics
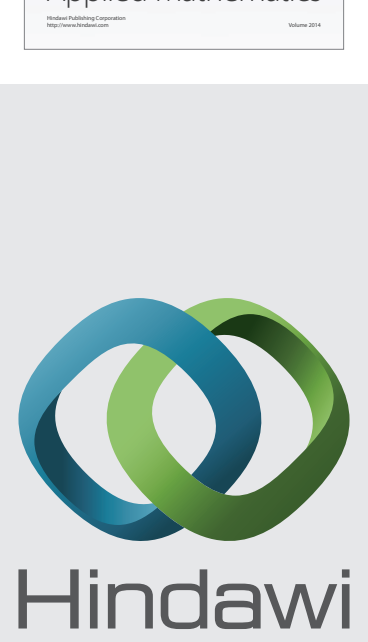

Submit your manuscripts at http://www.hindawi.com
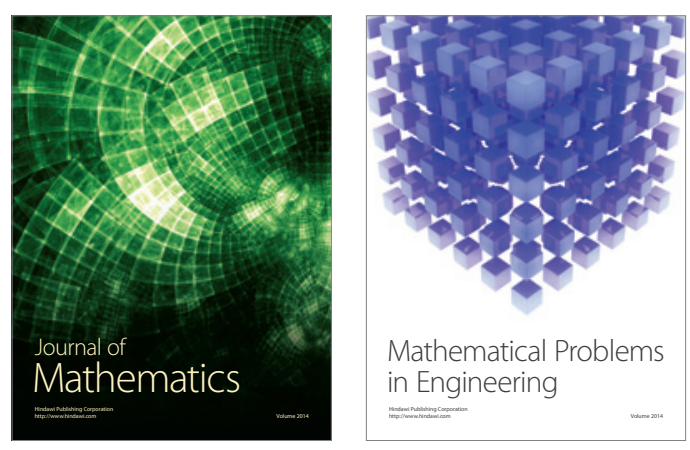

Mathematical Problems in Engineering
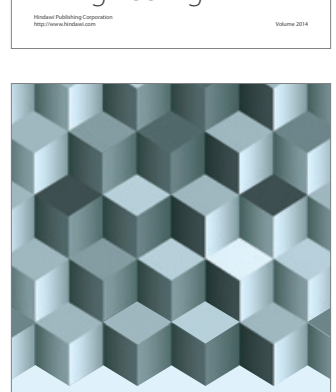

Journal of

Function Spaces
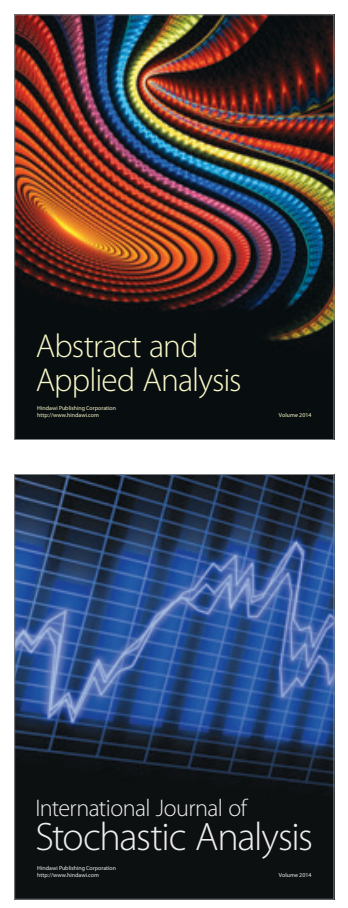

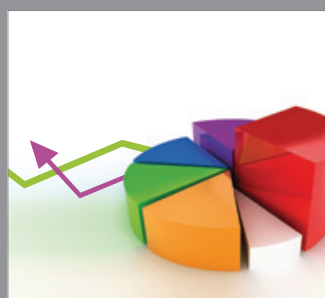

ournal of

Probability and Statistics

Promensencen
